\section{Institut \\ NeuroMyoGène : \\ un partenariat \\ franco-canadien \\ au service de la \\ recherche sur \\ les maladies \\ neuromusculaires}

\section{Laurent Schaeffer}

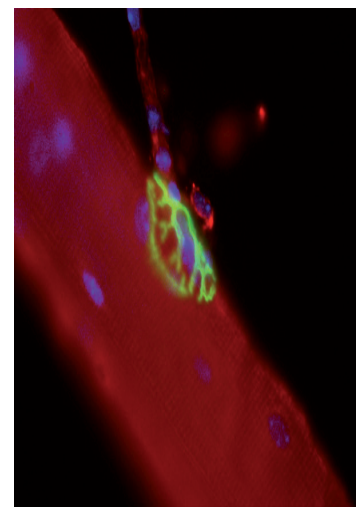

Directeur, Institut

NeuroMyoGene,

UMR 5310/U1217/UCBL,

Université Claude Bernard Lyon 1,

France.

laurent.schaeffer@ens-lyon.fr naire jusqu'au vieillissement. Ces approches sont rendues possibles grâce à un environnement extrêmement favorable regroupant 14 équipes de recherche de premier plan et des cliniciens renommés, dont les directeurs de trois centres de référence «maladies rares » dédiés aux maladies neuromusculaires, aux syndromes paranéoplasiques et aux maladies génétiques du rythme cardiaque.

La recherche à I'INMG est fortement soutenue par l'Association Française contre les Myopathies (AFM-Téléthon) qui permet à I'INMG d'être un acteur important de la structuration de la recherche française sur les maladies neuromusculaires au travers du réseau MyoNeurALP. À terme, I'INMG pourra accueillir jusqu'à 250 personnes dédiées à la recherche sur les maladies neuromusculaires.

$\varepsilon n$ vue de renforcer et d'étendre les liens existants aux chefs de file dans le domaine, I'INMG a récemment forgé un Accord de partenariat stratégique avec le Centre de recherche sur les maladies neuromusculaires de l'Université d'Ottawa (CRMN), à Ottawa au Canada. Créé en 1999, le mandat du CRMN est «d'améliorer la compréhension des MNM et des troubles connexes, et de promouvoir la santé par l'élaboration et la mise en œuvre de nouveaux traitements pour les MNM ».

Plus de quinze ans plus tard, le CRMN a connu une croissance remarquable et compte aujourd'hui plus de 25 équipes de spécialistes en recherche fondamentale et clinique ( 200 chercheurs et stagiaires), constituant l'une des plus importantes concentrations de chercheurs de calibre mondial dédié au domaine des MNM. Les membres du CRMN se sont illustrés partout dans le monde grâce à leur expertise en MNM pédiatriques et chez les adultes, y compris la maladie de Duchenne de Boulogne, la myotonie dystrophique, la maladie d'Aran-Duchenne, la sclérose latérale amyotrophique, les neuropathies périphériques, les syndromes myasthéniques congénitaux, la myasthénie grave et l'atrophie musculaire.

Une vision intégrée et concertée de la recherche, assortie d'une expertise complémentaire représente une occasion exceptionnelle de regrouper les chercheurs et les cliniciens du CRNM et de I'INMG avec le but commun d'accélérer la création de nouvelles connaissances fondamentales des MNM et de développer des approches thérapeutiques novatrices. L'accord de partenariat stratégique entre le CRMN et I'INMG soutiendra des domaines ciblés d'intérêt et d'expertise communs en vue de catalyser des initiatives de recherche concertées afin d'améliorer (la prise en charge et les stratégies thérapeutiques) pour les patients atteints de MNM. Ainsi, le CRMN et I'INMG créeront un programme de formation conjoint bilingue dédié aux MNM ouvert à 


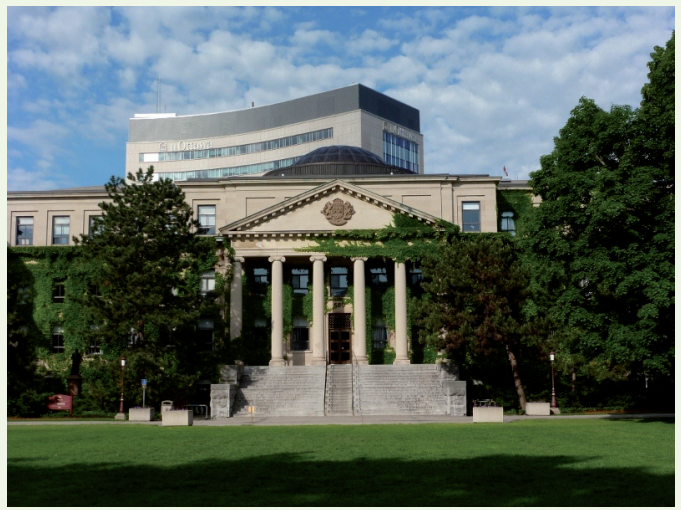

\section{同 uOttawa}

la prochaine génération de chercheurs et de cliniciens. Dans le cadre du programme de formation binationale, les stagiaires, les chercheurs principaux et les cliniciens seront en mesure de se déplacer librement entre les deux instituts. De plus, des vidéo-conférences et séminaires sur les travaux en cours seront prévus sur une base régulière. En dernier lieu, cette stratégie de recherche concertée permettra d'améliorer les initiatives conjointes de recherche en multipliant les possibilités de partage des échantillons de patients et de l'expertise technique en laboratoire, tout en augmentant la participation aux essais cliniques thérapeutiques.

Ce partenariat précieux et unique vient renforcer les efforts de recherche des deux instituts et fournira un milieu de recherche international, novateur, et productif pour former la prochaine génération de spécialistes en recherche fondamentale et clinique sur les MNM.

NeuroMyoGene Institute: a Franco-Canadian partnership promoting research in neuromuscular disorders

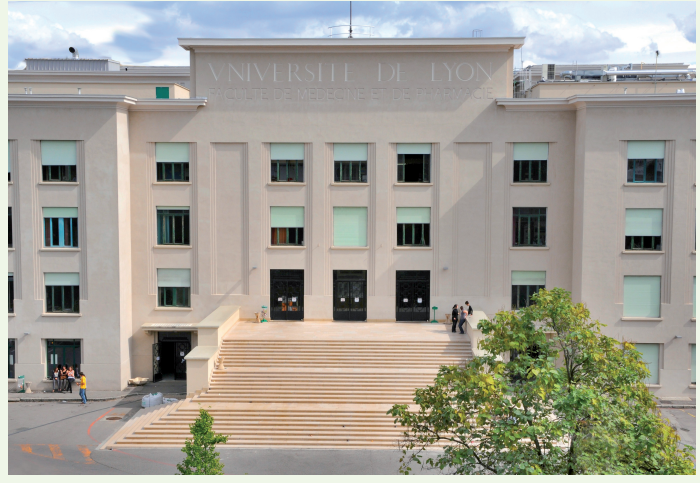

Université Claude Bernard Lyon 1

\section{REMERCIEMENTS}

L. Schaeffer remercie Mlle Charlene Clow ainsi que les Drs Bernard Jasmin, Rashmi Kothary, Robin Parks et Jodi Warman Chardon (membres du comité exécutif du CNMD à Ottawa) pour leurs contributions à l'établissement de ce partenariat et à l'écriture de ce document.

TIRÉS À PART

L. Schaeffer

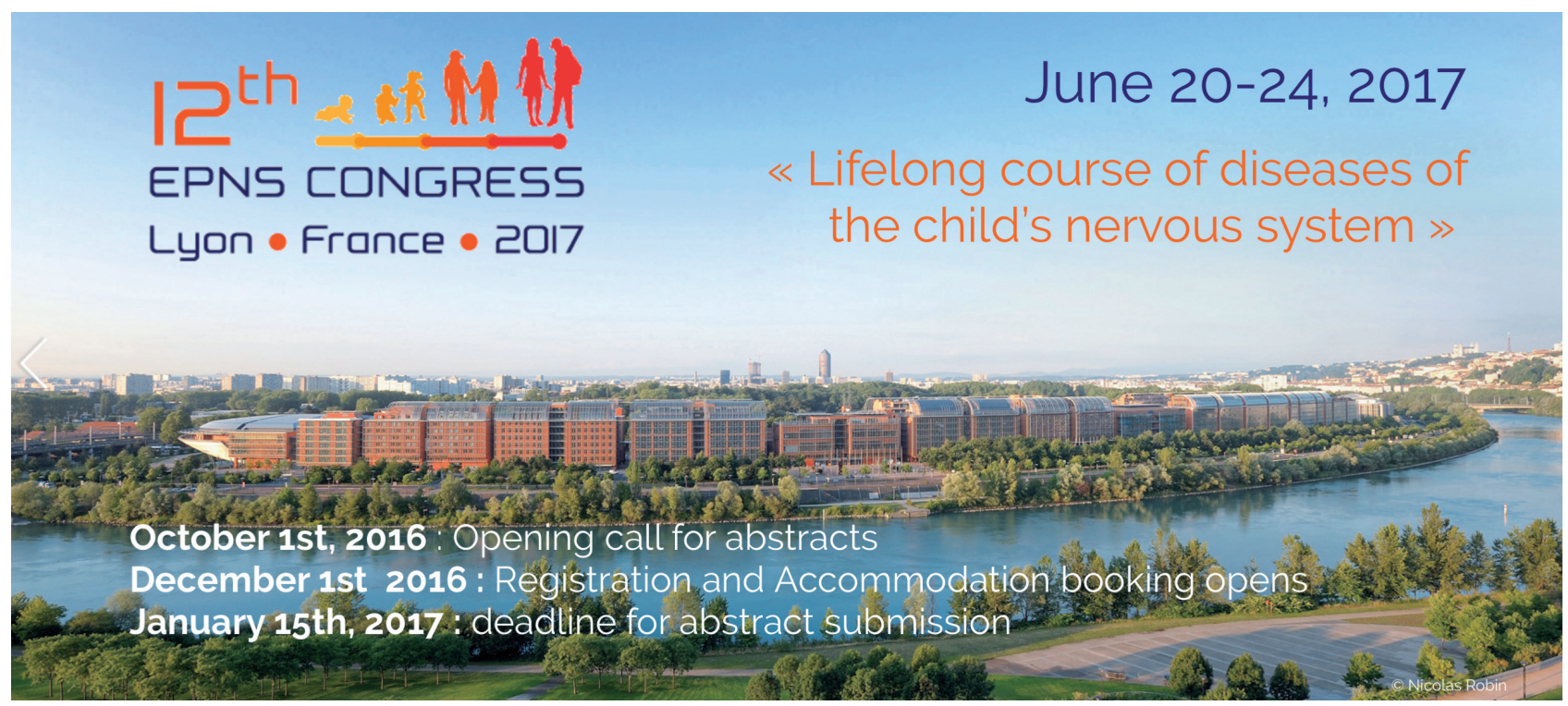

